\title{
Socioeconomic status inequity in inpatient service utilization based on need among migrants: Evidence from a national study in China
}

\section{Yi Wang}

School of Public Health, Cheeloo College of Medicine, Shandong University

Zhengyue Jing

Shandong University

Lulu Ding

Shandong University

Xue Tang

Shandong University

Yuejing Feng

Shandong University

Jie Li

Shandong University

Zhuo Chen

College of Public Health, University of Georgia

Chengchao Zhou ( $\nabla$ zhouchengchao@sdu.edu.cn )

Shandong University https://orcid.org/0000-0002-9364-3579

\section{Research}

Keywords: Migrants, socioeconomic status (SES), inpatient service utilization, concentration index, Blinder-Oaxaca decomposition, China

Posted Date: May 22nd, 2020

DOl: https://doi.org/10.21203/rs.3.rs-29742/v1

License: (c) (i) This work is licensed under a Creative Commons Attribution 4.0 International License.

Read Full License 

7

Yi Wang, Zhengyue Jing, Lulu Ding, Xue Tang, Yuejing Feng, Jie Li, Zhuo Chen, Chengchao

\section{Socioeconomic status inequity in inpatient service utilization based on need among}

$$
\text { migrants: Evidence from a national study in China }
$$

\section{Zhou $^{*}$}

Yi Wang; School of Public Health, Cheeloo College of Medicine, Shandong University, Jinan, 250012, China; wangyi031104@163.com Zhengyue Jing; School of Public Health, Cheeloo College of Medicine, Shandong

University, Jinan,250012, China; 2680929012@qq.com Lulu Ding; School of Public Health, Cheeloo College of Medicine, Shandong University, Jinan, 250012, China; 1531520959@qq.com

Xue Tang; School of Public Health, Cheeloo College of Medicine, Shandong University, Jinan, 250012, China; tessie0929@163.com Yuejing Feng; School of Public Health, Cheeloo College of Medicine, Shandong University, Jinan, 250012, China; fengyuejing1994@163.com Jie Li; School of Public Health, Cheeloo College of Medicine, Shandong University, Jinan, 250012, China; lijie2@126.com Zhuo Chen; College of Public Health, University of Georgia, Athens, GA 30606, USA; School of Economics, University of Nottingham, Ningbo, China, zchen1@uga.edu 


\section{Abstract}

Background: Equity in access to healthcare is a major health policy challenge in many lowand middle- income countries. However, millions of people, especially migrants, do not have the adequate access to health care they need. This study aims to identify the socioeconomic status (SES) inequities in inpatient service utilization based on need among migrants by using a nationally representative study in China.

Methods: The data used in this study was derived from the 2014 National Internal Migrant Population Dynamic Monitoring Survey collected by the National Health Commission of China. We used logistic regression method and Blinder-Oaxaca decomposition and calculated the concentration index to measure inequities of SES in inpatient service utilization based on need. Sample weights provided in the survey were applied in all the analysis to represent the China population.

Results: The total number of the migrants who needed inpatient service told by doctors was 7592, of which, 1667 (21.96\%) did not use the inpatient services (unmet inpatient service need). Results showed that inpatient service utilization concentrated among high-SES 
about $53.76 \%$ of the total SES gap in inpatient service utilization could be attributed to the gradient effect. After adjusting for other confounding variables, the odds ratios of inpatient service utilization by internal migrants with high SES according to educational attainment, economic status, and employment status were 1.41 (95\% CI 1.08-1.85, $p=0.012), 1.25$ (95\% CI $1.01-1.56, p=0.046)$, and $1.62(95 \%$ CI 1.12-2.36, $p=0.011)$, respectively.

Conclusion: This study observed an inequity in inpatient service utilization where the utilization concentrates among high SES migrants. This suggests that future policies should make the reimbursement more pro-poor among migrants in primary care and use more effective policies targeting the migrants with low educational attainment and unemployed, such as health education activities.

\section{Keywords}

Migrants, socioeconomic status (SES), inpatient service utilization, concentration index, Blinder-Oaxaca decomposition, China

\section{Background}

According to the World Health Organization (WHO) [1], the key goal of the universal health coverage (UHC) is to ensure that everyone receive the health care they need. Providing equal treatment for those who have the same need for healthcare, regardless of their socioeconomic and cultural background, has become a shared goal among policymakers who strive to improve healthcare. However, millions of people, especially migrants, do not have the adequate access to health-care services they need [1]. Migrants face many obstacles in accessing essential health care services due to factors such as language barriers, a lack of inclusive health policies, 
and inaccessible public services [2]. The WHO has been promoting the health of migrants and committed to adequately address health needs for migrants. A WHO framework for migrant health has recognized the urgent need for the health sector to address the impact of migration on health effectively [2].

China has experienced the largest migration during the past three decades, with the number of migrants increased from 230 million in 2011 to 244 million in 2017, which constituted $18 \%$ of the total population of China [3]. Internal migrants, in Chinese literally "floating population", which is defined as those who have left their hometowns to live and work in a new place for more than one month but do not have a local 'Hukou' (registered residence) at the new location [4]. Since 1980s, the rate of urbanization has increased dramatically in China which is due to internal rural-to-urban migration [5]. However, migrants are known to be marginalized in China, because of the Hukou system. Although the internal migrants have made an important contribution to urban economic growth and social stability, their health status and health service utilization have not received due attention. Comparing with local residents, the migrants face many barriers in accessing essential health care, which lead to unmet health care need and poor quality care $[2,6]$. The challenge for China is how to promote economic growth and develop wealth while reducing inequality among migrants. Addressing the health care needs of migrants can improve their health status, facilitate social integration, and contribute to economic development [7]. During the past two decades, China implemented several national healthcare development plans [8] to improve healthcare access and equality with many challenges and successes. National Health Commission of the People's Republic of China (NHC-PRC) has started an initiative called 'Equal Access to Public Services among 
Migrants (EAPSM)' since 2013, to improve access to public service especially the utilization of health service.

According to previous studies, socioeconomic status (SES) has been found to be a key determinant in the utilization of health services. A study in Korea found that women with lower SES have limited access to necessary health care service [9]. Another study conducted in China showed that the patients with higher SES preferred public health services than private health services [10]. A few studies also explored the needs and utilization of health services among migrants. A study [11] in the United States identified factors related to healthcare utilization among Asian migrant women and found that those had health insurance and more work-related health symptoms were more likely to visit a primary care provider. Guo and colleagues examined potential linkages between family relationships and health service utilization among Chinese American elderly, which showed that positive spousal or family relations were not associated with either physician visits or hospital stays [12]. Another study [13] in China found that migrants who were insured were more likely to have doctor visits than those who were uninsured. These existing studies have mostly been divided into three categories. The first is about the difference and comparison of the utilization of health services between the migrants and the local residents $[6,14,15]$. Second, most studies on the internal migrants are based on regional data [13, 16-19], and there were few studies using a nation-wide data about the migrants. More importantly, most of the studies focused on the influencing factors of the utilization of health services of the migrants, but few explored from the perspective of health need $[18,20,21]$. Equity in health can only be attained if persons with the same level of healthcare needs receive equal level of care, regardless of their socioeconomic status. 
111 Although health equity became an increasingly popular research topic worldwide, there is a

112 shortage of studies explored the SES inequities in inpatient service utilization among internal

113 migrants in China, especially based on need. It is also essential for more studies to evaluate

114 the situation in the utilization of health services after EAPSM. In order to fill these gaps in the

115 current studies, this study was performed to explore the SES inequities in inpatient service

116 utilization based on need among the internal migrants in China, in order to quantify SES roles

117 in healthcare utilization inequity as a guide for health policy makers and draw public policy

118 implications to further reform the health care systems.

\section{Methods}

\section{Study design and data}

122 The data used in this study was derived from the 2014 National Internal Migrant Population by the National Health Commission of China. The purpose of the survey was to investigate the utilization of health services among internal migrants. The sampling frame for this study was taken using the stratified multistage random sampling method by probability proportional to size (PPS) approach. All respondents in this study were aged 15-59 years who had been living in local residence without the 'Hukou' for more than one month, including migrants from both rural and urban areas. For more details on sampling, design and approvals of the survey, please refer to an earlier study. [23] The detailed sampling process was shown in Fig. 1. Finally, a total of 7592 migrants with inpatient service need were included in this nationally representative analysis. 
134 In this study, migrants' inpatient service need was measured by questions about whether they

135 were asked to be hospitalized by a doctor during the last 12 month (inpatient need). Based on

136 the inpatient service need, the outcome was categorized into unmet inpatient services need and

137 receiving inpatient services. The unmet need for inpatient service referred to the proportion

138 migrants who were asked to be hospitalized but did not utilize it. The key independent variable

139 was socioeconomic status (SES). SES was an economic and sociological combined total

140 measure of a person's work experience and an individual's or family's economic and social

141 position in relation to others, based on household income, individual education, and

142 employment status [24]. In this study, we assessed the SES in two ways. First, in order to

143 compare the inequity of the high-low SES in inpatient service utilization from a macro

144 perspective, we integrated the educational level, economic status (household income per

145 month) and employment status into a single SES index using principal component analysis

146 (PCA) [25] method (details see in Appendix Table A1). Then, we used three specific

147 indicators (economic status, employment status and educational attainment) to show the

148 associations between SES and inpatient service utilization. All three socioeconomic status

149 types were measured in two categories: low SES and high SES. Low and high SES was

150 defined in the following ways: 1) educational attainment, as middle school or below vs. high

151 school or above; 2) economic status was created using a median split based on household

152 income per month; 3) employment status which means whether the respondent had a job, as

153 employed vs. unemployed. 

utilization, controlled variables included gender, age, marital status (married or single), number

156 of children and ethnic group (Han or ethnic minority), whether had a health record, Hukou

157 types (urban or rural), health insurance, movement area (across province, city or county),

158 duration of migration, region, and willingness for long-term residence of more than 5 years (yes,

159 no, and not decided yet). Types of health insurance were divided into five subgroups: no health

160 insurance, having New Rural Cooperative Medical Scheme (NCMS), having Urban Employee

161 Basic Medical Insurance (UEBMI) and having Urban Resident Basic Medical Insurance

162 (URBMI). Movement area was categorized into three types: migration across provinces;

163 migration across prefectural cities but within a province and migration across counties but

164 within a prefectural city. All the controlled variables were available through the 2014 National

165 Internal Migrant Population Dynamic Monitoring Survey and were included in multivariate

166 logistic regression model 2.

\section{Analytical methods}

168 Data analyses were conducted by using the STATA 14.2. Descriptive analyses were performed

169 to compare the inpatient service utilization across different subgroups of the participants using

$170 \mathrm{t}$-test or chi-square test as appropriate and reported their $p$-values. Sample weights were

171 applied in all the analysis to represent the China population.

172 First, we estimated the concentration index (CI) and constructed a concentration curve

173 (CC) to illustrate inequity in unmet inpatient service need among migrants. The CC graphs the

174 cumulative percentage of the sample on the x-axis, ranked by SES index, beginning with the

175 lowest. CI was used to quantify the magnitude of inequity in unmet need and corresponds to 
twice the area between the $\mathrm{CC}$ and the $45^{\circ}$ line [29]. CC runs from -1 (over-diagonal) to +1 (under-diagonal), indicating whether the unmet inpatient service need is concentrated among the low-SES $(\mathrm{CI}<0)$, the high-SES $(\mathrm{CI}>0)$, or equally distributed $(\mathrm{CI}=0)[30]$.

The concentration index $C_{M}$ was calculated by the following formula:

$$
=\frac{2}{\bar{y}} \operatorname{cov}_{w}\left(y_{i}, R_{i}\right)
$$

Where $\bar{y}$ stands for the mean of $y, y_{i}$ is the measure of unmet inpatient service need of $i$ th individual, $R_{i}$ denotes the fractional rank of the $i$ th individual in the SES index, and $\operatorname{cov}_{w}$ is the covariance with sampling probability weights. The concentration index and the associated $p$-values were obtained by the delta method [31]. If the $C_{M}$ is significantly smaller than 0 , low SES individuals are more likely to have unmet inpatient service need, and vice versa [32].

Then, we adopted logistic regression method to investigate the SES disparities in multivariate analyses adjusted for confounding variables. Those who received inpatient services were defined as the reference group. Binary logistic regression (model 1) to examine the association between SES and inpatient service utilization without controlled variables. In order to control for potential confounding factors, multiple logistic regression (model 2) were used to estimate the adjusted odds ratio and the $95 \%$ confidence intervals. The model was specified as:

$$
\begin{gathered}
\operatorname{Logit}\left(\frac{p_{i}}{1-p_{i}}\right)=\beta_{0}+\beta_{1} * \mathrm{SES}_{\mathrm{i}}+\beta_{2} * \mathrm{C}_{\mathrm{i}}+\epsilon \\
\text { OR }=\operatorname{Exp}\left(\beta_{1}\right)
\end{gathered}
$$


Coefficients $\beta_{0}$ and $\beta_{1}$ represented intercept and SES inequalities, respectively; $\epsilon$ indicated error terms; OR indicated Odds Ratio.

SES migrants was assessed using the Blinder-Oaxaca (BO) decomposition method. The BO decomposition method was originally developed to explain wage gaps between whites and blacks and between men and women since the seminal work of Oaxaca and Blinder in the early 1970s [33, 34]. The BO decomposition [35]was a counterfactual method with an assumption that "what the probability of unmet inpatient service need would be if low SES 207 migrants had the same characteristics as their high SES counterparts". In this part, SES was created using a median split with low SES categorized as below the median of SES index total score and high SES categorized as above the median. Based on it, the SES inequity was divided into two parts by using $\mathrm{BO}$ decomposition as followed:

214 coefficients. The first term in Equation (1) corresponded to the proportion of the gap in

215 outcomes between two groups that were accounted for by group differences in the distribution

216 of observable characteristics, it indicated "endowments effect"; while the second term was

217 "gradient effect" traces the differences that are attributable to the effect of the variables.

218 Decomposing SES differences in inpatient service utilization into endowments and gradient 
effects has strong policy implications since the evidence of gradient effect would reflect that

high-low SES migrants endowed with the same characteristics do not enjoy the same level of

221 inpatient service.

\section{Results}

According to the Table 1, the total number of the migrants who needed inpatient service diagnosed by doctors was 7592, of which, 1667(21.96\%) did not use the inpatient services (unmet inpatient service need) and 5925(78.04\%) had used the inpatient services. The migrants with high-SES defined by educational attainment, economic status, and employment status was 2942 (which accounted for 38.75\% in total), 3438 (45.28\%), and 7137 (94.01\%), respectively. Of the 7592 participants, about two-thirds $(n=5461)$ were female. The mean age was $32(\mathrm{SD}=9.02)$ years old. Most of the migrants were Han Chinese and had married, $97.43 \%$ had at least one child; $82.86 \%$ were registered as having a rural 'Hukou' and $74.39 \%$ had established the health records in the local residence. Overall, $58.57 \%$ of the respondents were covered by the NCMS, $7.26 \%$ and $20.13 \%$ were covered by the URBMI and UEBMI, respectively, while $14.04 \%$ had no social health insurance. Generally speaking, the majority of our sample population was migrants across province (48.08\%) and has willingness for long-term residence (66.81\%). Using chi-square tests, we found that there were statistically

237 significant differences in socioeconomic status, gender, age, marital status, number of children, 238 health insurance, movement area, and duration of migration. Figure 2 plotted the concentration curves for probability of inpatient service utilization among migrants in the 
previous 12 month. A significant distribution of inpatient service utilization based on need concentrated among high-SES migrants was observed (CI: 0.041, $P<0.001)$.

In Table 2, we showed the association between SES and those who receive inpatient disparities in utilization of inpatient services in different socioeconomic status without covariate adjustment. The OR values of inpatient service utilization by internal migrants with high SES according to educational attainment, economic status, and employment status, which were 1.54 (95\% CI 1.23-1.93, $p<0.001), 1.39$ (95\% CI 1.14-1.71, $p=0.001)$, and 2.24 (95\% CI $1.60-3.14, p<0.001)$, respectively. After adjusting for other confounding variables such as gender, age, marital status etc., the associations were still statistically significant in model 2. according to educational attainment, economic status, and employment status were 1.41 (95\% CI 1.08-1.85, $p=0.012), 1.25$ (95\% CI 1.01-1.56, $p=0.046)$, and 1.62 (95\% CI 1.12-2.36, $p=0.011)$, respectively. Logistic regression analysis showed that migrants with higher SES defined by educational attainment were 1.41 times, economic status were 1.25 times, and employed migrants were 1.62 times than the lower SES to utilize the inpatient healthcare services. Figure 3 showed the composition of the reasons for unmet inpatient service need, of unnecessary $(574,34 \%)$. 
endowments effect and gradient effect were significant in logistic decompositions, $46.24 \%$ of the gap between the two groups could be attributed to differences in the distribution of explanatory variables included in the model. About $53.76 \%$ of the total SES difference in inpatient service utilization could be attributed to gradient effect.

\section{Discussion}

268 Healthcare utilization based on need is a key indicator to assess the operation of a country's

269 healthcare system, and any barriers of access to healthcare should be identified and then

270 eliminated [9]. It is important to assess equity in meet health services need rather than access

271 to healthcare, since access simply denotes an opportunity to receive healthcare, while meeting

272 need mean utilizing the opportunity. One of the objectives of UHC is equity in access to

273 healthcare services, which means 'everyone who needs these services should get them, not

274 just those who can pay for them'[36]. By analyzing the SES inequities in inpatient service

275 utilization based on need among the migrants is vital to develop targeting measures, so as to

276 better meet the health services need of the migrants. The present study can also be considered

277 as the first results of evaluating the situation in utilization of inpatient services after EAPSM.

278 Using the National Internal Migrant Population Dynamic Monitoring Survey dataset in 2014,

279 we found that the rate of unmet inpatient service need among migrants was $22.75 \%$, which

280 was higher than $17.1 \%$ of general population [37], implying the migrants still face many

281 barriers in accessing essential health care than the local residents. CI has been widely used in

282 the health inequity literature. This study found that CI was significantly larger than 0 and the

283 CC lying over the line of equality, meaning inpatient service utilization concentrated more 
among the high SES group. Socioeconomic inequality in the use of healthcare, i.e., the high

SES group having a higher probability of healthcare utilization when needed, is a persistent in low- and middle- income countries [38]. Our results are similar to previous studies on general healthcare utilization in China $[39,40]$.

Logistic regression was used to show the association between SES and inpatient service utilization. We found the three SES indicators, including economic status, employment status and educational attainment, were statistically significant. Our study indicated that low economic status of internal migrants was a key barrier to accessing inpatient service. Compared with those in the low-economic status group, internal migrants with higher economic status were more likely to utilize inpatient service when they had an inpatient service need, which was consistent with the top reason shown in Figure 3 (economic hardship, 36.29\%) for unmet inpatient service need among internal migrant. Previous studies have shown that the risk of unmet inpatient service of the poor people was significantly higher than that of non-poor people[27], both in the permanent residents and the migrants[4, 41]. There are several possible reasons for this finding. First, migrants with higher economic status in China have higher payment capacity, and hence, they were more likely to use inpatient services when in need. Second, most of those with low economic status were those rural-to-urban migrants. The primary goal of migration among this population was in search of economic opportunities in urban areas. Thus, they tended to focus on their economic conditions only, and usually do not prioritize their own health [2]. Even if they needed inpatient health services, going to hospital would cost them a fortune. Despite the nearly universal medical insurance coverage in China, economic status remains the dominant barrier to healthcare services utilization [28, 42, 43], 
including outpatient and inpatient services, and lead to inequity in general health care utilization[44-46]. This phenomenon is even more severe among the internal migrants. This study also found that low educational attainment was associated with unmet inpatient service need among internal migrants, which was consistent with other studies [20, 47-49]. One possible interpretation for this finding was that the internal migrants with higher education usually received more knowledge and awareness about the importance of inpatient service use, and thus tended to use inpatient services when they have a need. The present study revealed

313 that employed migrants were more likely to use inpatient service when needed than the

314 unemployed migrants, which was consistent with previous studies [20]. The employed migrants with formal job usually conduct regular medical examinations and receive health

316 education from their work place. In contrast, most of the unemployed migrants might be a

317 dependent of the migrant who had a job, which result in less health education. In addition, 318 unemployed migrants have no financial source, thus, one another possible interpretation for 319 this finding was the inability of unemployed migrants to meet the costs. These reasons may 320 explain the lower possibility to use inpatient service when they in need concentrated among 321 unemployed migrants. probability of inpatient service utilization and the gradient effect, rather than the endowments

324 effect, accounted for most of the SES difference in unmet inpatient service need. In other words, about $46.24 \%$ gap in inpatient service utilization between low and high SES can be explained by difference in the levels of observable characteristics. The "gradient effect",

327 which was considered as "discrimination" in previous studies, could reflect inequity here. The 
decomposition results suggested that about $53.76 \%$ of the total SES gap in inpatient service utilization could be attributed to the gradient effect. Namely, SES inequity could account for around 54\% in inpatient service utilization among migrants. Migrants with lower SES may choose to delay or resist the need of inpatient services since meeting the need of inpatient services often means high medical expenses. Improving social and economic resources of low SES migrants would be helpful for reducing the barriers of unmet inpatient need. To be specific, policy makers should develop pro-poor health insurance scheme in migrants with low economic status and those who unemployed. Also, future interventions might consider using health education focused on migrants with low level of education. It is worth mentioning that popular and easy ways should be conducted to intervene for migrants with low educational attainment and improve their use of inpatient service when in need. For example, a better form of health education on migrants is peer education. Those low education migrants with similar age profile, gender and economic status can have common topics of discussion, and thus share information, so as to amplify the effect of "peer effect".

Although previous studies have shown that high-SES is a protective factor in using public health service among the migrants $[10,17,20,21,50,51]$, few explored from the perspective of health need among internal migrants in China. As to study limitation, it should be noted that the utilization of inpatient services and doctor's diagnostic information of the internal migrants were both self-reported, therefore, recall bias might exist. Second, due to the lack of the information on the use of outpatient services, we cannot analyze the utilization of full health services of migrants. 


\section{Conclusion}

351 This study observed an inequity in inpatient service utilization where the utilization

352 concentrates among high SES migrants. To be specific, three individual-level SES indicators

353 of internal migrants including economic status, employment status, and educational

354 attainment were all significantly associated with inpatient service utilization. Internal

355 migrants with higher economic status, higher educational attainment and employed were

356 more inclined to utilize inpatient services when needed. This suggests that future policies

357 should make the reimbursement more pro-poor among migrants in primary care and more

358 effective policies targeting the migrants with low educational attainment and unemployed,

359 such as health education activities.

\section{List of abbreviations}

363 SES: socioeconomic status; UHC: universal health coverage; WHO: World Health

364 Organization; NHC-PRC: National Health Commission of the People's Republic of China;

365 EAPSM: Equal Access to Public Services among Migrants; PPS: probability proportional to

366 size; PCA: Principal Component Analysis; NCMS: New Rural Cooperative Medical Scheme;

367 UEBMI: Urban Employee Basic Medical Insurance; URBMI: Urban Resident Basic Medical

368 Insurance; CI: Concentration Index; CC: Concentration Curve; BO: Blinder-Oaxaca; OR:

Odds Ratio 
373 Not applicable.

\section{Consent for publication}

375 Not applicable.

\section{Availability of data and materials}

377 The datasets are open to all of the potential users online.

378 [http://www.chinaldrk.org.cn/wjw/\#/data/classify/population/yearList].

\section{Competing interests}

380 The authors declare that they have no competing interests.

$381 \quad$ Funding

382 This study was supported by the National Science Foundation of China (Grant Numbers

383 71974117, 71473152, 71774104), the China Medical Board (16-257), Cheeloo Youth Scholar

384 Grant, and Shandong University (IFYT1810, IFYT181031). The funding bodies had no role

385 in the design, data collection, analysis, interpretation of the data, and writing of this article.

\section{Authors' contributions}

$387 \mathrm{CCZ}$ and ZC conceived the idea and polished the manuscript. YW coded and analyzed data

388 and wrote the manuscript. ZJ, YF, XT, and LD participated in interpretation of the data. All

389 authors read and approved the final manuscript.

390 Acknowledgements

391 This research uses data from the National Internal Migrant Population Dynamic Monitoring

392 Survey. We thank the National Health Commission of China providing this data available

393 online. 
396 References

397 1. World Health Day 2019. World Health Organization; 2019.

398 2. Zheng L, Hu R, Dong Z, Hao Y: Comparing the needs and utilization of health services 399 between urban residents and rural-to-urban migrants in China from 2012 to 2016. BMC $400 \quad$ Health Serv Res 2018, 18:717.

$401 \quad 3 . \quad$ China Statistical Yearbook. National Bureau of Statistics 2018.

402 4. Peng B, Zou G, Chen W, Lin Y, Ling L: Association between health service utilisation 403 of internal migrant children and parents' acculturation in Guangdong, China: a $404 \quad$ cross-sectional study. Bmj Open 2018, 8:e018844.

405 5. Yu C, Lou C, Cheng Y, Cui Y, Lian Q, Wang Z, Gao E, Wang L: Young internal 406 migrants' major health issues and health seeking barriers in Shanghai, China: a 407 qualitative study. BMC Public Health 2019, 19:336.

408 6. Gu H, You H, Ning W, Zhou H, Wang J, Lu Y, Sun J, Kou Y, Dong H: Internal 409 migration and maternal health service utilisation in Jiangsu, China. Trop Med Int $410 \quad$ Health 2017, 22:124-132.

411 7. International Migration, Health and Human Rights. 2013.

412 8. Views on promoting progressive equalization of basic public health services. The 413 Central People's Government of the People's Republic of China; 2009.

$4149 . \quad$ Hwang J: Understanding reasons for unmet health care needs in Korea: what are health 415 policy implications? BMC Health Serv Res 2018, 18:557. 
416 10. Wang Q, Zhang D, Hou Z: Insurance coverage and socioeconomic differences in patient choice between private and public health care providers in China. Soc Sci Med 2016, 170:124-132.

11. Seo JY, Chao YY, Yeung KM, Strauss SM: Factors Influencing Health Service Utilization Among Asian Immigrant Nail Salon Workers in the Greater New York City Area. J Community Health 2019, 44:1-11.

12. Guo M, Sabbagh Steinberg N, Dong X, Tiwari A: Is family relations related to health service utilisation among older immigrants: Evidence from Chinese elderly in the

14. Wang Q: Health of the Elderly Migration Population in China: Benefit from Individual and Local Socioeconomic Status? Int J Environ Res Public Health 2017, 14.

16. Zou G, Zeng Z, Chen W, Ling L: Self-reported illnesses and service utilisation among

15. Lu CH, Luo ZC, Wang JJ, Zhong JH, Wang PX: Health-related quality of life and health service utilization in Chinese rural-to-urban migrant workers. Int J Environ Res Public Health 2015, 12:2205-2214. 
18. Zeng J, Zou G, Song X, Ling L: Contraceptive practices and induced abortions status among internal migrant women in Guangzhou, China: a cross-sectional study. $B M C$ Public Health 2015, 15:552.

19. An F, Broadbent K, Yuan F: Employment inequality among women migrant workers in China: comparative analysis from the 2014 Guangdong Migrant Workers Survey. Asia Pacific Journal of Human Resources 2018, 56:518-538.

20. Zhang J, Lin S, Liang D, Qian Y, Zhang D, Hou Z: Public Health Services Utilization and Its Determinants among Internal Migrants in China: Evidence from a Nationally Representative Survey. Int J Environ Res Public Health 2017, 14.

21. Song X, Zou G, Chen W, Han S, Zou X, Ling L: Health service utilisation of rural-to-urban migrants in Guangzhou, China: does employment status matter? Trop Med Int Health 2017, 22:82-91.

22. China Migrants Dynamic Survey. Migrant Population Service Center of Nation Health Commission P.R.China; 2015.

23. Wang H, Zhang D, Hou Z, Yan F, Hou Z: Association between social health insurance and choice of hospitals among internal migrants in China: a national cross-sectional study. BMJ Open 2018, 8:e018440.

24. Pampel FC, Krueger PM, Denney JT: Socioeconomic Disparities in Health Behaviors. Annu Rev Sociol 2010, 36:349-370.

25. Abolfazl, Abouie, Reza, Majdzadeh, Roghayeh, Khabiri, Soudabeh, Hamedi-Shahraki, Seyed, Hasan: Socioeconomic inequities in health services' utilization following the Health Transformation Plan initiative in Iran. Health Policy \& Planning 2018, 0:1-8. 
26. Fleurbaey M, Schokkaert E: Unfair Inequalities In Health And Health Care. Journal of Health Economics 2009, 28:73-90.

27. Wang N, Shi S, Fan J, Zhu Y: Unused inpatient service and its influencing factors of residents in Henan Province. Journal of zhengzhou university (medical science) 2016,

28. Zhu D, Guo N, Wang J, Nicholas S, Chen L: Socioeconomic inequalities of outpatient and inpatient service utilization in China: personal and regional perspectives. Int $J$ Equity Health 2017, 16:210.

29. Watanabe R, Hashimoto H: Horizontal inequity in healthcare access under the universal coverage in?Japan;?1986-2007. Social Science \& Medicine 2012, 75:1372-1378.

30. Zhuo C, Roy K: Calculating concentration index with repetitive values of indicators of economic welfare. Journal of Health Economics 2009, 28:0-175.

31. Koolman X, Doorslaer E: On the interpretation of a concentration index of inequality. Health Economics 2004, 13:649-656.

32. Eiko S, Stuart G, Daisuke Y, Shyam GG, Mizanur RM, Krishna SP, Kenji S: Inequality and inequity in healthcare utilization in urban Nepal: a cross-sectional observational study. Health Policy \& Planning 2016, 7:1-8.

33. Blinder AS: Wage Discrimination: Reduced Form and Structural Estimates. Journal of Human Resources 1973, 8:436-455. Economic Review 1973, 14:693. 
35. Kelishadi R, Qorbani M, Heshmat R, Djalalinia S, Sheidaei A, Safiri S, Hajizadeh N, Motlagh ME, Ardalan G, Asayesh H, Mansourian M: Socioeconomic inequality in childhood obesity and its determinants: a Blinder-Oaxaca decomposition. J Pediatr (Rio J) 2018, 94:131-139.

36. Health Financing for Universal Coverage. Geneva: World Health Organization. World Health Organization; 2014.

37. Xu 1, Meng q: Results of the fifth national health service survey ii - health service needs, needs and utilization (in Chinese). Chinese journal of health information management 2014:193-194.

38. Peltzer K, Williams JS, Kowal P, Negin J, Snodgrass JJ, Yawson A, Minicuci N, Thiele L, Phaswana-Mafuya N, Biritwum RB, et al: Universal health coverage in emerging economies: findings on health care utilization by older adults in China, Ghana, India, Mexico, the Russian Federation, and South Africa. Glob Health Action 2014, 7:25314.

39. Gabriel, M., Leung, Keith, Y., K., Tin, Owen, O'Donnell: Redistribution or horizontal equity in Hong Kong's mixed public-private health system: a policy conundrum. Health Economics 2009, 18:37-54.

40. Elwell-Sutton TM, Jiang CQ, Zhang WS, Cheng KK, Lam TH, Leung GM, Schooling CM: Inequality and inequity in access to health care and treatment for chronic conditions in China: the Guangzhou Biobank Cohort Study. Health Policy \& Planning $2012,5: 5$

41. Glaesmer H, Wittig U, Braehler E, Martin A, Mewes R, Rief W: Health care utilization among first and second generation immigrants and native-born Germans: a 
population-based study in Germany. Int J Public Health 2011, 56:541-548.

42. Wang Y: Growing old before growing rich: inequality in health service utilization among the mid-aged and elderly in Gansu and Zhejiang Provinces, China. Bmc Health Services Research 2012, 12:302.

43. Zhang X, Zhao L, Cui Z, Wang Y: Study on Equity and Efficiency of Health Resources and Services Based on Key Indicators in China. PLoS One 2015, 10:e0144809.

44. Elwell-Sutton TM, Jiang CQ, Zhang WS, Cheng KK, Lam TH, Leung GM, Schooling $\mathrm{CM}$ : Inequality and inequity in access to health care and treatment for chronic conditions in China: the Guangzhou Biobank Cohort Study. Health Policy Plan 2013, $28: 467-479$.

45. Zhang X, Wu Q, Shao Y, Fu W, Liu G, Coyte PC: Socioeconomic inequities in health care utilization in China. Asia Pac J Public Health 2015, 27:429-438.

46. Guan M: Should the poor have no medicines to cure? A study on the association between social class and social security among the rural migrant workers in urban China. Int J Equity Health 2017, 16:193.

47. Guo J, Wen HY, Zhou QY: Status quo and determinants on basic public health services of floating population. Chinese Journal of Health Policy 2014:51-56(Chinese).

48. Tian M, Wang H, Tong X, Zhu K, Zhang X, Chen X: Essential Public Health Services' Accessibility and its Determinants among Adults with Chronic Diseases in China. Plos One 2015, 10:e125262.

49. Lu L, Zeng J, Zeng Z: What limits the utilization of health services among china labor force? analysis of inequalities in demographic, socio-economic and health status. Int $J$ 
527 50. Shao S, Wang M, Jin G, Zhao Y, Lu X, Du J: Analysis of health service utilization of migrants in Beijing using Anderson health service utilization model. BMC Health Serv Res 2018, 18:462.

530 51. Zhang R, Li S, Li C, Zhao D, Guo L, Qu P, Liu D, Dang S, Yan H: Socioeconomic inequalities and determinants of maternal health services in Shaanxi Province, Western China. PLoS One 2018, 13:e0202129.

Table 1 Characteristics of the migrants who need to be hospitalized, China $(n=7592)$

\begin{tabular}{|c|c|c|c|c|}
\hline \multirow[t]{2}{*}{ Characteristics } & \multirow[t]{2}{*}{ Total N (\%) } & \multicolumn{3}{|c|}{ Inpatient services } \\
\hline & & Receive N (\%) & Unmet need N (\%) & $p$-value \\
\hline Total & 7592 & $5925(78.04)$ & 1667 (21.96) & \\
\hline \multicolumn{5}{|l|}{ Socioeconomic status } \\
\hline Educational attainment & & & & $<0.001$ \\
\hline High & $2942(38.75)$ & $2479(41.84)$ & $463(27.77)$ & \\
\hline Low & $4650(61.25)$ & $3446(58.16)$ & $1204(72.23)$ & \\
\hline Economic status & & & & $<0.001$ \\
\hline High & $3438(45.28)$ & $2838(47.90)$ & $600(35.99)$ & \\
\hline Low & $4154(54.72)$ & $3087(52.10)$ & $1067(64.01)$ & \\
\hline Employment status & & & & $<0.001$ \\
\hline
\end{tabular}




\begin{tabular}{|c|c|c|c|c|}
\hline Employed & $7137(94.01)$ & $5659(95.51)$ & $1478(88.66)$ & \\
\hline Unemployed & 455 (5.99) & $266(4.49)$ & $189(11.34)$ & \\
\hline \multicolumn{5}{|l|}{ Controlled variables } \\
\hline Gender & & & & $<0.001$ \\
\hline Female & $5461(71.93)$ & $4670(78.82)$ & $791(47.45)$ & \\
\hline Male & $2131(28.07)$ & $1255(21.18)$ & $876(52.55)$ & \\
\hline Age & $32.97 \pm 9.02$ & $31.48 \pm 8.15$ & $38.27 \pm 9.91$ & $<0.001$ \\
\hline Marital status & & & & $<0.001$ \\
\hline Married & $7120(93.78)$ & $5649(95.34)$ & $1471(88.24)$ & \\
\hline Single & $472(6.22)$ & $276(4.66)$ & $196(11.76)$ & \\
\hline Number of children & & & & $<0.001$ \\
\hline 0 & $195(2.57)$ & $126(2.13)$ & $69(4.14)$ & \\
\hline 1 & $4070(53.61)$ & $3340(56.37)$ & $730(43.79)$ & \\
\hline$\geqq 2$ & $3327(43.82)$ & $2459(41.50)$ & $868(52.07)$ & \\
\hline Ethnic group & & & & 0.91 \\
\hline Han & $6915(91.08)$ & $5396(91.07)$ & $1519(91.12)$ & \\
\hline Ethnic minority & $677(8.92)$ & $529(8.93)$ & $148(8.88)$ & \\
\hline Health records & & & & 0.47 \\
\hline Yes & $5648(74.39)$ & 4396 (74.19) & $1252(75.10)$ & \\
\hline No & $1944(25.61)$ & $1529(25.81)$ & $415(24.90)$ & \\
\hline Hukou & & & & 0.061 \\
\hline
\end{tabular}




\begin{tabular}{|c|c|c|c|c|}
\hline Urban & $1301(17.14)$ & $1041(17.57)$ & $260(15.60)$ & \\
\hline Rural & $6291(82.86)$ & $4884(82.43)$ & $1407(84.40)$ & \\
\hline Health insurance & & & & 0.002 \\
\hline No insurance & $1066(14.04)$ & $844(79.17)$ & $222(20.83)$ & \\
\hline NCMS & 4447 (58.57) & $3433(77.20)$ & $1014(22.80)$ & \\
\hline URBMI & $551(7.26)$ & $410(74.41)$ & $141(25.59)$ & \\
\hline UEBMI & $1528(20.13)$ & $1238(81.02)$ & $290(18.98)$ & \\
\hline Movement area & & & & 0.034 \\
\hline Across province & $3650(48.08)$ & 2867 (48.39) & $783(46.97)$ & \\
\hline Across city & $2330(30.69)$ & $1838(31.02)$ & $492(29.51)$ & \\
\hline Across county & $1612(21.23)$ & $1220(20.59)$ & $392(23.52)$ & \\
\hline Migration time (year), & $4.51 \pm 4.80$ & $4.09 \pm 4.42$ & $6.03 \pm 5.73$ & $<0.001$ \\
\hline \multicolumn{3}{|c|}{ Plans for long-term residence( $>5$ years) } & & 0.093 \\
\hline Yes & $5072(66.81)$ & $3940(66.50)$ & $1132(67.91)$ & \\
\hline No & $740(9.75)$ & $565(9.54)$ & $175(10.50)$ & \\
\hline Not decided yet & $1780(23.45)$ & $1420(23.97)$ & $360(21.60)$ & \\
\hline
\end{tabular}

536 Note: Hukou refers to the household registration system in China, classified all residents into

537 rural and urban registration categories;

538 NCMS: New Rural Cooperative Medical Scheme; UEBMI: Urban Employee Basic Medical

539 Insurance; URBMI : Urban Resident Basic Medical Insurance.

540 
542 Table 2 Association between socioeconomic status and receivers of inpatient services among

543 migrants who need them, China $(\mathrm{n}=7592)$

\begin{tabular}{lccc}
\hline Characteristics & Model 1 & Model 2 \\
\cline { 2 - 3 } & Unadjusted OR $(95 \% \mathrm{CI})$ & $p$-value & Adjusted OR $(95 \% \mathrm{CI}) \quad p$-value
\end{tabular}

\section{Socioeconomic status}

Educational attainment

\begin{tabular}{|c|c|c|c|}
\hline High & $1.54(1.23,1.93)$ & $<0.001$ & $1.41(1.08,1.85)$ \\
\hline Low & Ref. & & Ref. \\
\hline
\end{tabular}

Economic status

\begin{tabular}{|c|c|c|c|}
\hline High & $1.39(1.14,1.71)$ & 0.001 & $1.25(1.01,1.56)$ \\
\hline Low & Ref. & & Ref. \\
\hline
\end{tabular}

Employment status
High
$2.24(1.60,3.14)$
$<0.001$
$1.62(1.12,2.36)$
0.011
Low
Ref.
Ref.

Note: Sample weights applied; CI indicated confidence interval; Model 2 were adjusted for

545 gender, age, marital status, number of children, ethnic group, health record, Hukou type,

546 health insurance, movement area, duration of migration and willingness for long-term

547 residence of more than 5 years. 
Table 3 BO decomposition of the gap in inpatient service utilization when needed between

551 the high and low SES among migrants in China $(\mathrm{n}=7592)$

\begin{tabular}{lllll}
\hline & Coef. $(95 \% \mathrm{CI})$ & SE & Contrib. $(\%)$ & $\mathrm{P}$ \\
\hline Predicted probability & & & & \\
High SES & $0.854(0.834,0.873)$ & 0.010 & $<0.001$ \\
Low SES & $0.761(0.738,0.784)$ & 0.012 & - & $<0.001$ \\
Difference in predicted & & & & \\
probability & & & & $<0.001$ \\
Total gap & & & & \\
Due to endowments effect & $0.043(0.027,0.060)$ & 0.008 & 46.24 & $<0.001$ \\
$\quad$ & $0.050(0.019,0.080)$ & 0.015 & 53.76 & $<0.001$ \\
\hline Due to gradient effect & & 0.015 & 100 & \\
\hline
\end{tabular}

552 Note: Regressions and decompositions are weighted with sampling weights provided in the

553 survey.

554

$555 \quad$ Figures

556 Figure 1: Flow chart of sample selection

557 Figure 2: Concentration curves for probability of inpatient service use among migrants

558 Figure 3: Reasons for unmet inpatient service need among the migrants 
Figures

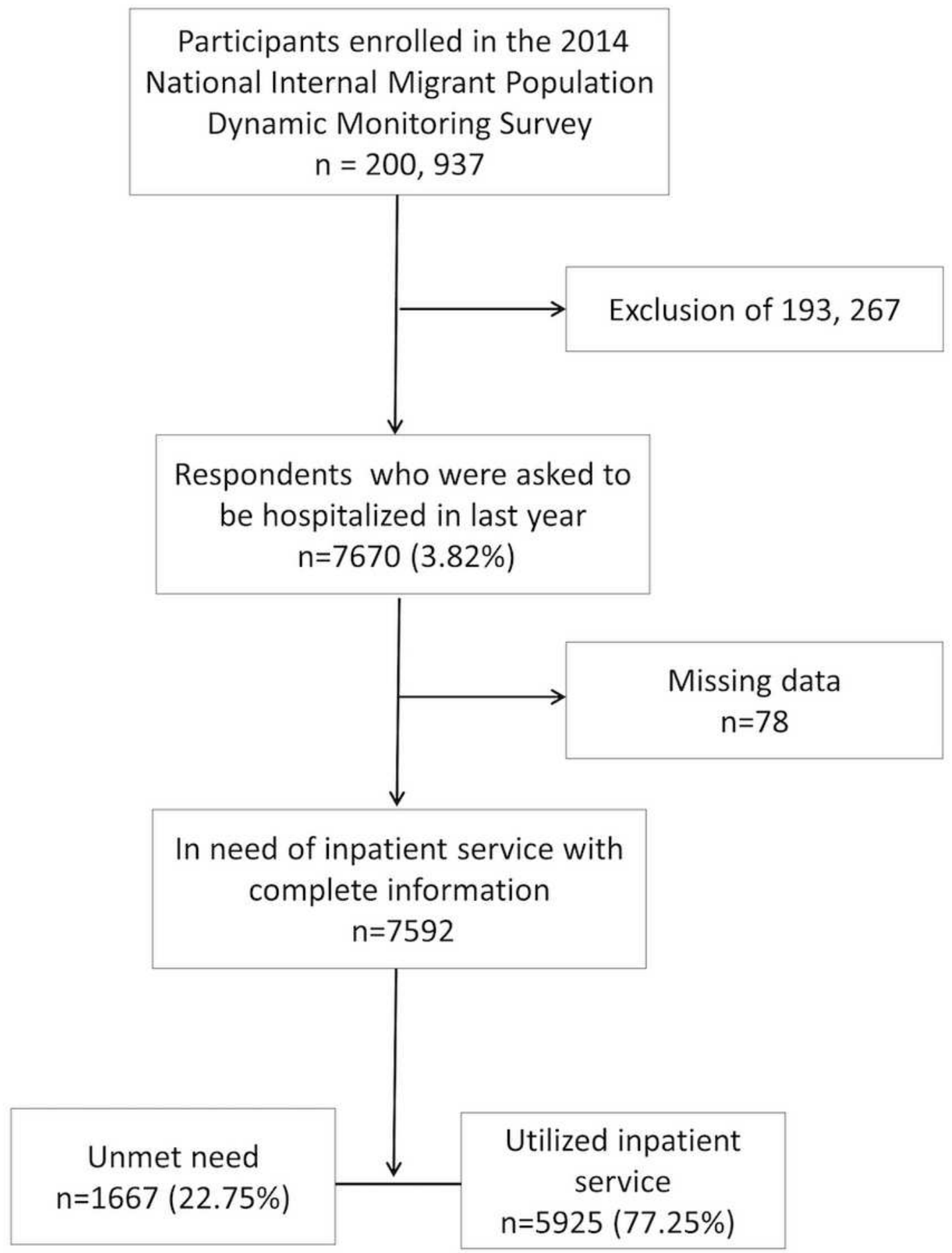

Figure 1

Flow chart of sample selection 


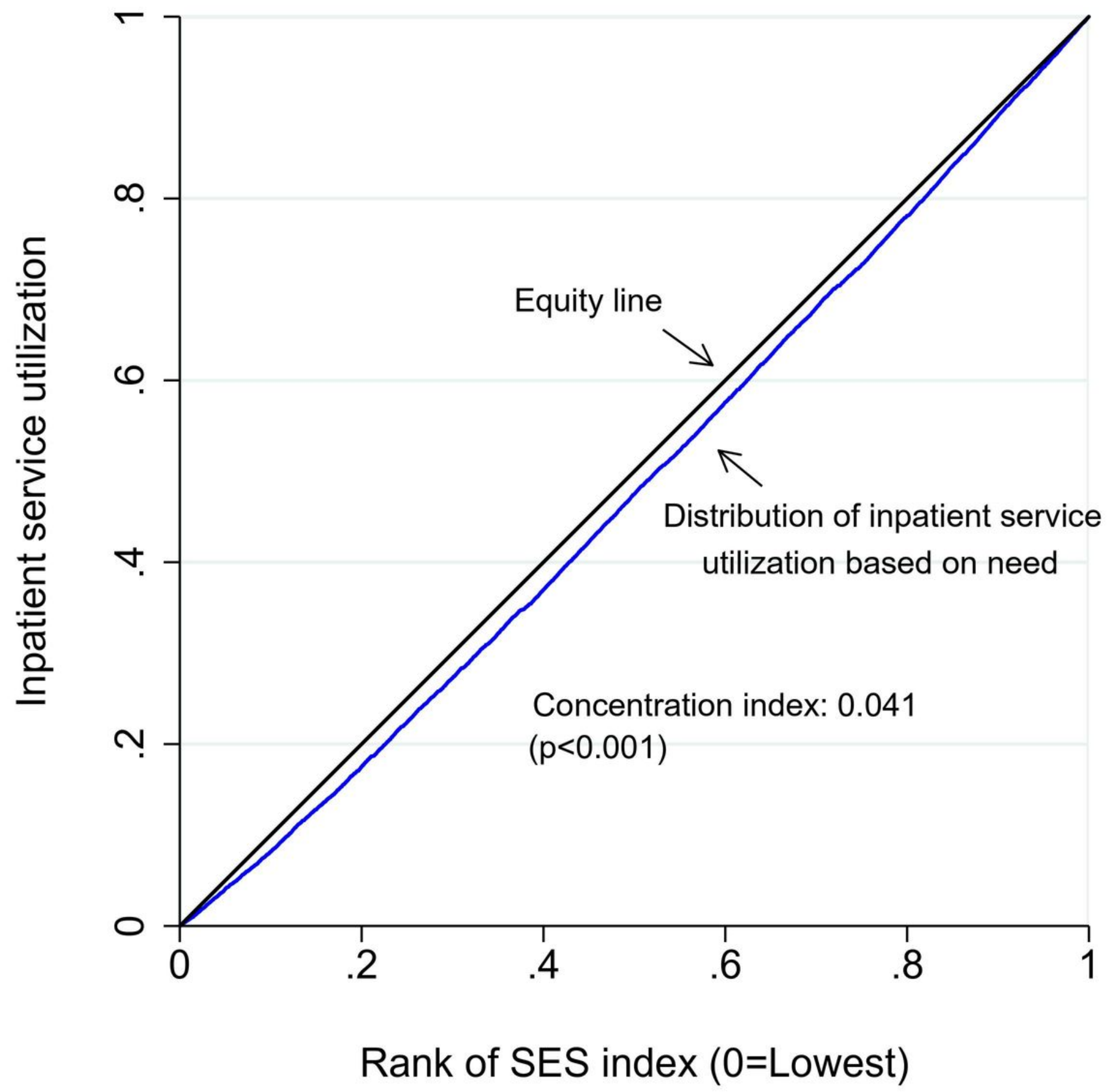

Figure 2

Concentration curves for probability of inpatient service use among migrants 


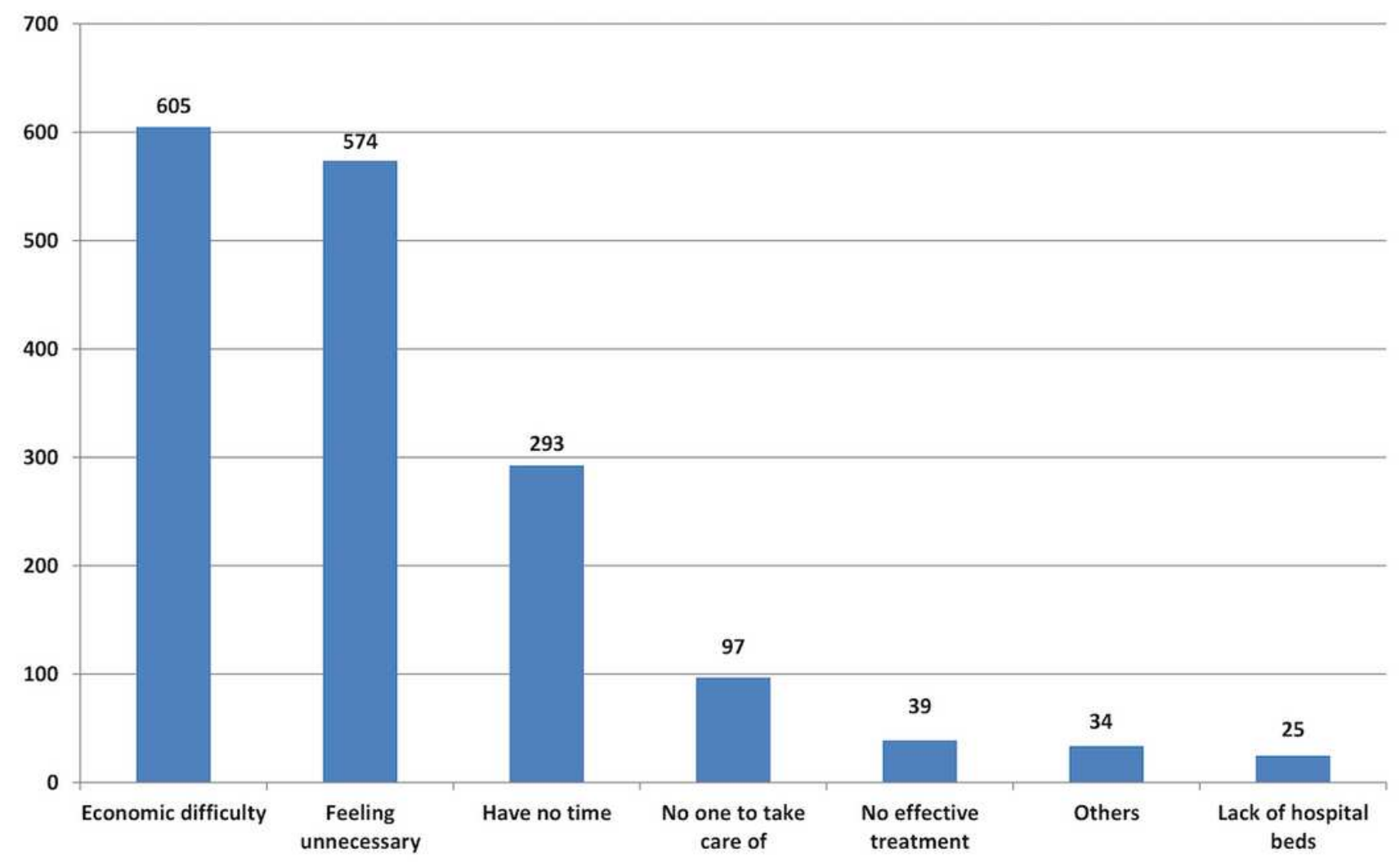

Figure 3

Reasons for unmet inpatient service need among the migrants

\section{Supplementary Files}

This is a list of supplementary files associated with this preprint. Click to download.

- SupplementaryAppendixTableA1.docx

- SupplementaryAppendixTableA1.docx 